\title{
Nuclear expression of survivin portends a poor prognosis in Merkel cell carcinoma
}

\author{
Jinah Kim and Jennifer M McNiff \\ Department of Dermatology, Yale University School of Medicine, New Haven, CT, USA
}

\begin{abstract}
Inhibition of apoptosis is a critical step in tumorigenesis in many cancers, including Merkel cell carcinoma; however, the exact regulatory mechanisms are not fully understood. Survivin is an inhibitor of apoptosis that is undetectable in most terminally differentiated normal human tissues, strongly expressed in embryonic and fetal organs and is strongly expressed in many different human cancers. In this study, we investigated the expression of survivin in cutaneous Merkel cell carcinoma using immunohistochemistry and correlated the findings with long-term clinical follow-up. We collected and immunostained 19 cases of Merkel cell carcinoma with antibodies to survivin. The median patient age was 79 years, with an average follow-up of 17 months, and a male/female ratio of 7:11. All but one sample represented primary lesions and two cases were obtained from one patient. Clinical follow-up was obtained in 15 cases (79\%). All 19 cases of Merkel cell carcinoma demonstrated strong immunoreactivity for survivin. Survivin protein was localized and classified into predominately nuclear $(N=8)$ or cytoplasmic $(N=4)$ compartments. A mixed pattern of survivin expression was also seen in three cases. Cases with a nuclear staining pattern were distinguished by an aggressive clinical course, with seven of eight patients developing metastases or dead of disease on follow-up. Furthermore, all of the cases with predominately cytoplasmic survivin localization $(N=4)$ were free of disease on follow-up. Merkel cell carcinomas represent aggressive malignancies regulated by apoptotic pathways. We demonstrate that survivin, a protein with a dual role in inhibition of apoptosis and regulation of cellular proliferation is expressed in Merkel cell carcinoma. Moreover, nuclear subcellular localization of survivin in Merkel cell carcinomas may portend a poor prognosis and identification of these cases may assist clinical management. Modern Pathology (2008) 21, 764-769; doi:10.1038/modpathol.2008.61; published online 18 April 2008
\end{abstract}

Keywords: survivin; Merkel cell carcinoma; prognosis; apoptosis

In 1875 Merkel described a unique epidermal cell that he believed to be a special sensory cell of the skin. ${ }^{1}$ These cells are thought to be the mechanoreceptors of the basal layer of the epidermis and may interact with terminal axons. Merkel cells are presumed to originate in the neural crest and are characterized by co-expression of neuronal and epithelial markers. ${ }^{2}$ Merkel cell carcinoma is an uncommon malignant epithelial cutaneous neoplasm with neuroendocrine differentiation. In 1972 Toker first described five cases of 'trabecular carcinoma of the skin'. ${ }^{3}$

Seen predominately in elderly Caucasians $(0.23$ annual age-adjusted incidence per 100000$),{ }^{4}$ approximately 1500 new cases of Merkel cell carcinoma occur each year. ${ }^{5}$ Merkel cell carcinoma often presents as a painless firm rapidly growing

Correspondence: Dr J Kim, MD, PhD, Department of Dermatology, Yale University School of Medicine, 15 York Street, LMP 5031, New Haven, CT 06510, USA.

E-mail: jinah.kim@yale.edu

Received 16 December 2007; revised and accepted 27 February 2008; published online 18 April 2008 violaceous papule or plaque that is usually present on sun-exposed areas, with greater than $50 \%$ of the cases on the head and neck, $40 \%$ on the extremities and fewer than $10 \%$ on the trunk and genitalia. ${ }^{6}$ Accordingly, ultraviolet radiation and immunosuppression have been demonstrated to have etiologic roles. Merkel cell carcinomas are considered neuroendocrine neoplasms with cytologic features including distinct nuclear membrane, finely dispersed chromatin with scant cytoplasm. Histologically, the tumors may have a diffuse, nested, or trabecular growth pattern.

Merkel cell carcinomas are aggressive tumors with a high incidence of local recurrence and lymph node and distant metastasis. ${ }^{7}$ The 5 -year cause-specific survival is $58 \%$ for Stage I and $38 \%$ for Stage II disease. ${ }^{8}$ Clinical factors that have been determined to portend a poor prognosis include the following: advanced age $>55$ years, location on the head and neck, size greater than $2 \mathrm{~cm}$, presence of immunosuppression and advanced stage of disease. ${ }^{6,7,9} \mathrm{In}$ addition, histological factors that portend a poor prognosis include a high level of mitoses, small cell size, and angiolymphatic invasion. ${ }^{10}$ Also, recently 
p63 immunostaining has been shown to be an independent prognostic marker for aggressive Merkel cell carcinomas. ${ }^{11}$

In the epidermis, keratinocytes rely heavily on apoptosis for normal development and to promote the steady turnover that permits the elimination of premalignant cells. In contrast, inhabitants of the epidermis that do not need to be constantly 'turned over' have been demonstrated to require antiapoptotic strategies to maintain longevity. The cost of this longevity is the accumulation of defects that may progress to carcinoma. Melanomas and Merkel cell carcinomas utilize altered anti-apoptotic mechanisms for tumor survival and this fact is postulated to be one cause of chemotherapeutic failure. Therefore, we sought to better understand the role of apoptosis in the regulation of Merkel cell carcinoma growth.

Apoptosis occurs by two main pathways: (1) death receptors (Fas and TNFR1), and (2) mitochondrial integrity (cytochrome $c$ ). Both pathways converge on the activation of a family of intracellular serine proteases, or caspases, to dismantle the energy production and cellular architecture. Bcl-2 protein, which is located on the outer mitochondrial membrane, blocks apoptosis by inhibition of cytochrome $C$ release. Interestingly, BCL2 is highly expressed in Merkel cell carcinoma and suppression of Bcl-2 protein expression through the use of antisense oligonucleotides has been shown to partially suppress Merkel cell carcinoma growth in a murine model. ${ }^{12}$ Inhibition of mitochondrial apoptosis may also occur through a second pathway that acts at a step downstream of $B C L 2$ that involves the family of inhibitors of apoptosis. ${ }^{13}$ Members of the inhibitor of apoptosis gene family prevent the proteolytic maturation and activation of caspases to regulate apoptosis.

Survivin is a $16.5 \mathrm{kDa}$ intracellular protein that contains a domain characteristic of the inhibitors of apoptosis family, the baculovirus IAP repeat domain, and functions to prevent the proteolytic maturation and activation of caspases. ${ }^{14}$ Survivin is a unique inhibitor of apoptosis in that it has been shown to be undetectable in most terminally differentiated normal human tissues, strongly expressed in embryonic and fetal organs, ${ }^{15,16}$ and strongly expressed in many different human cancers, including melanoma. ${ }^{13,17}$ Therefore, we sought to determine whether there are other antiapoptotic pathways that are important in the regulation of Merkel cell carcinoma growth.

\section{Materials and methods}

A total of 19 cases of Merkel cell carcinoma identified from archival specimens at Yale University Dermatopathology Laboratory comprise this study. The hematoxylin and eosin stained sections and immunoperoxidase preparations were re- viewed. The diagnosis of Merkel cell carcinoma was confirmed by immunohistochemical methods; all cases demonstrated paranuclear dot-like immunoreactivity for cytokeratin 20 and the absence of TTF1 immunoreactivity.

\section{Immunohistochemistry}

Formalin-fixed paraffin-embedded tissue sections were immunostained for survivin. Sections $4 \mu \mathrm{m}$ thick were deparaffinized in xylene, rehydrated in alcohol, and blocked for endogenous activity (3\% $\mathrm{H}_{2} \mathrm{O}_{2}$ and normal rabbit serum). Antigen retrieval was performed by steaming in high $\mathrm{pH}$ buffer. The sections were incubated overnight with the polyclonal antibody AB469 (Abcam Inc., Cambridge, MA, USA) at a dilution of 1:400 for all cases. Two separate investigators were blinded to the original samples and independently evaluated the intensity, localization and pattern of survivin expression. Cases were considered positive if greater than $75 \%$ of the tumor cells demonstrated immunoreactivity. The subcellular expression pattern was evaluated and characterized as (1) nuclear, (2) cytoplasmic, or (3) mixed nuclear and cytoplasmic compartments. A threshold value of $>75 \%$ was established for a positive result. We then obtained clinical follow-up to determine whether there was a correlation between the pattern of survivin expression by immunoperoxidase staining and prognosis.

\section{Results}

\section{Clinical Findings}

Of the 19 cases collected, 18 were from separate patients (Table 1). The average age at diagnosis was 79 years with a range from 50 to 88 years. The male: female ratio was $7: 11$. The lesions were present of the head and neck in 11 cases, the extremity in five cases, and the chest and buttock in one case each. The majority of patients were with stage I disease with only one patient each with stage II and III disease.

We were able to obtain clinical follow-up on 15 of the 19 cases $(79 \%)$ with an average follow-up period of 17 months (range, 4-31 months) (Table 1). The immunoperoxidase results demonstrated eight of 15 cases $(53 \%)$ with clinical follow-up had a strong predominately nuclear pattern of survivin immunoreactivity (Figure 1a and b). In addition, three of these eight cases $(38 \%)$ with a nuclear distribution of survivin were found to be dead of disease on clinical follow-up (Table 1). In addition, another four cases $(50 \%)$ with nuclear survivin staining were shown to have distant metastasis (breast, brain, lung, abdomen and soft tissue) on clinical followup. Only one case $(13 \%)$ with a predominant nuclear staining pattern was free of disease with a follow-up period of 18 months. In contrast, all the 
Table 1 Clinical demographics and survivin staining pattern in merkel cell carcinoma

\begin{tabular}{lcccllll}
\hline Case number & Stage & Age & Sex & Location & Follow-up & Length of follow-up (mo) & Survivin staining pattern \\
\hline 1 & I & 78 & F & Right eyebrow & Lost & N/A & Cytoplasmic \\
2 & I & 84 & F & Left 3rd finger & Lost & N/A & Cytoplasmic \\
3 & I & 76 & M & Buttock & Lost & N/A & Cytoplasmic \\
4 & I & 85 & M & Right cheek & Lost & 9 & Cytoplasmic \\
5 & I & 86 & F & Right neck & DOD & 14 & Nuclear \\
6 & II & 70 & M & Left neck & DOD & 4 & Nuclear/cytoplasmic \\
7 & I & 76 & M & Left cheek & DOD & Cytoplasmic \\
8 & I & 50 & F & Right cheek & NED & 30 & Nuclear \\
9 & I & 83 & F & Left arm & NED & 18 & Nuclear \\
10 & I & 84 & F & Right cheek & METS & 31 & Nuclear/cytoplasmic \\
11 & I & 82 & M & Chest & NED & 26 & Nuclear/cytoplasmic \\
12 & I & 88 & F & Right arm & NED & 31 & Cytoplasmic \\
13 & I & 73 & F & Left cheek & NED & 21 & Nuclear \\
14 & I & 71 & M & Scalp & METS & 6 & Cytoplasmic \\
15 & I & 83 & F & Left ankle & NED & 5 & Nuclear \\
16 & I & 71 & M & Scalp & METS & 18 & Nuclear \\
17 & I & 84 & M & Left ear helix & DOD & 19 & Cytoplasmic \\
19 & I & 87 & F & Right arm & NED & Nuclear
\end{tabular}

DOD, dead of disease; METS, metastasis; NED, no evidence of disease.
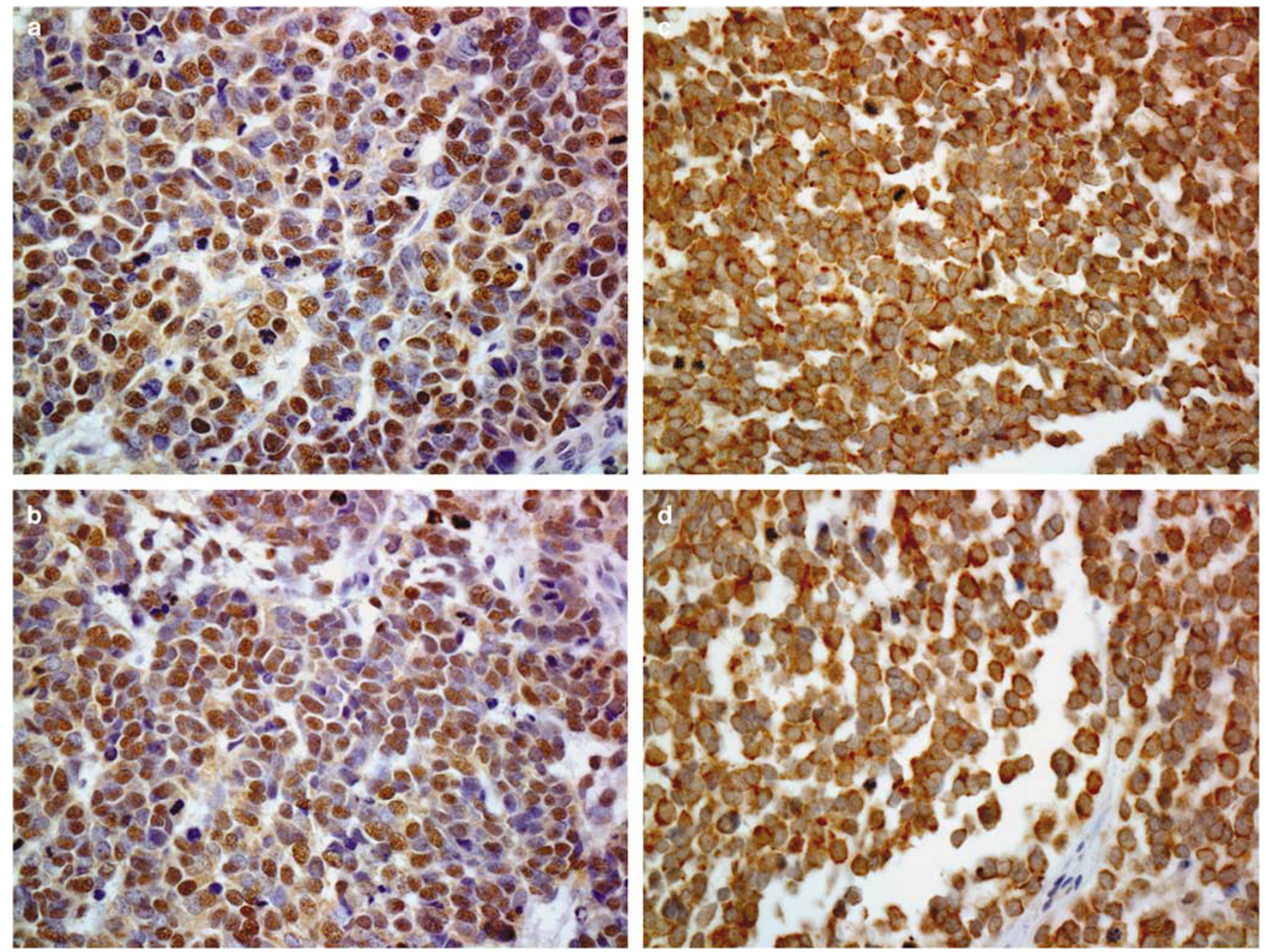

Figure 1 Immunohistochemical staining for survivin expression in Merkel cell carcinoma. (a and b) Survivin localization in the nuclear compartment. (c and d) Survivin localization in the cytoplasmic compartment. Original magnification $\times 200$. 
cases $(N=4)$ with predominant cytoplasmic pattern of survivin staining had on follow-up no evidence of disease with an average follow-up period of 25 months (Figure 1c and d). Therefore, in our study the nuclear pattern of survivin immunostaining appeared to correlate with a poor prognosis resulting in mortality or metastasis in the majority of cases (Table 1).

Immunostaining with p63 and Ki-67 (MIB-1) were also performed to determine whether nuclear localization of survivin in Merkel cell carcinoma correlated with an increased mitotic index or p63 staining, which have been shown to be negative prognostic factors in Merkel cell carcinoma. ${ }^{10,11}$ Ki-67 and p63 immunostaining were evaluated and classified into low $(<33 \%)$, moderate $(33-67 \%)$, or high $(>67 \%)$ categories based on the percentage of cells with positive immunostaining in our cases of Merkel cell carcinoma (data not shown). Low Ki-67 immunostaining was identified in seven cases, ${ }^{1-5,7,8}$ moderate staining was identified in five cases, ${ }^{9,10,15,17,18}$ and high staining was present in seven cases. ${ }^{6,11-14,16,19}$ In addition, an extremely high Ki-67 immunostaining pattern was identified in two cases ${ }^{14,19}$ which either clinically presented with soft tissue metastasis or lymphovascular invasion and metastasis. Immunostaining with p63 was negative in eight cases, ${ }^{1-4,9,13,17,19}$ low in four cases, ${ }^{12,14-16}$ moderate in two cases, ${ }^{11,18}$ and high in three cases. ${ }^{5,6,10}$ Two cases $^{14,16}$ demonstrated strong, though focal, p63 expression that was preferentially expressed in the lymphatic spaces and absent in the nodular tumor component.

\section{Discussion}

Survivin is truly a unique protein because it is ubiquitously expressed in embryonic and fetal tissue and is undetectable in most terminallydifferentiated human tissue. ${ }^{15,16}$ In addition, high expression of survivin is identified in numerous human malignancies, including melanoma, ${ }^{17}$ lung, ${ }^{18}$ breast, ${ }^{19}$ gastric, ${ }^{20}$ pancreatic, ${ }^{21}$ colon, ${ }^{22}$ uterine, ${ }^{23}$ ovarian, ${ }^{24}$ and bladder ${ }^{25}$ carcinomas.

The differential subcellular compartmentalization of survivin has been described in many human tumors and has also correlated with prognosis. Nuclear expression of survivin has been correlated with poor survival in cutaneous melanoma; high nuclear expression of survivin resulted in a decreased 5-year survival (53.3\%), as compared to low nuclear expression of survivin $(90.4 \%){ }^{26}$ Likewise, cases of non-small-cell lung carcinoma with nuclear survivin expression demonstrated a decreased overall survival $(20 \%)$, compared to cases in which tumors expressed cytoplasmic survivin $(44.5 \%){ }^{27}$

Originally described as a cytoplasmic anti-apoptotic protein that is overexpressed in numerous human malignancies, survivin has a dual role in the regulation and integration of apoptosis and mitosis.
In addition to its role in apoptosis, survivin has been shown to have a role in regulation of cell proliferation and cell viability. ${ }^{28}$ Survivin exists in immunohistochemically distinct subcellular microdomains within mitotic cells. ${ }^{29}$ In the nucleus, survivin is a member of the chromosomal passenger complex, which is comprised of Aurora B kinase, and its regulatory subunits inner centromeric protein, and Borealin. $^{30}$ Targeting of Aurora B kinase to the specific substrate at the proper time is essential for cell division. ${ }^{31}$ Survivin has been shown to associate with the chromosomal passenger complex and colocalize to the centromeres, spindle midzone, and midbody during metaphase, anaphase, and telophase, respectively. ${ }^{29,30}$ We observed co-localization of nuclear survivin with the mitotic spindle apparatus (Figure 2). In addition, survivin has been recently shown to be an essential protein in mitosis and mutation of a single threonine residue (T117) results in prometaphase arrest. ${ }^{32}$

In our study of 19 cases of Merkel cell carcinoma, all of the cases demonstrated expression of survivin. The intriguing aspect of this study is the differential subcellular localization of survivin into either nuclear or cytoplasmic compartments. Cases with nuclear survivin immunoreactivity on follow-up were found to be more likely to evolve into metastatic disease $(50 \%)$ or actually result in mortality in $38 \%$ of cases. Only one case with predominately nuclear staining on clinical follow-up had no evidence of disease; however, the follow-up period was only 18 months. In contrast, cases that demonstrated cytoplasmic survivin immunoreactivity had a better prognosis and all of these cases had no evidence of disease at the time of clinical follow-up (average period $=25$ months). In our results, the co-localization of nuclear survivin with the spindle apparatus also lends further biologic support of the role of survivin in regulation of Merkel cell

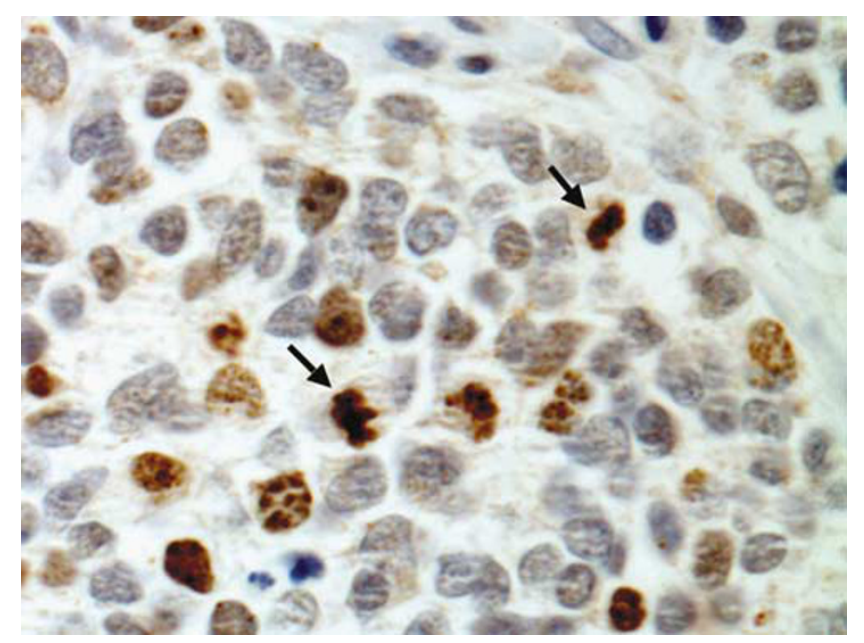

Figure 2 Immunohistochemical staining for survivin appears to co-localize with mitotic spindle in nuclear compartment of Merkel cell carcinoma (arrow). Original magnification $\times 200$. 
carcinoma proliferation. Although limited by small study number and short clinical follow-up period, our results suggest that nuclear survivin expression may portend a poorer prognosis in Merkel cell carcinoma.

In addition, small cell size and mitotic index have been reported to be histologic indicators of poor prognosis. ${ }^{10}$ We discovered that in one particular case with a bimorphic population of separate small and large cell foci, the larger cell type preferentially expressed survivin in the cytoplasmic compartment (Figure 3a and b). The correlation of the subcellular distribution of survivin localization by immunohistochemistry with a specific morphologic indicator of prognosis supports the results of our clinicopathologic study. The specific expression pattern within a single tumor may represent separate foci evolving into a higher grade or metastatic lesion. The identification of tumors with evolving potential for increased aggressive behavior will be of critical clinical significance and will undoubtedly improve clinical management.

Immunostaining with p63 was performed on 17 of 19 cases $(89 \%)$ with available tissue and was present in nine of 17 cases (53\%) with moderate to high p63 immunostaining observed in five of 17 cases (29\%). Nuclear or mixed (nuclear and cytoplasmic) survivin immunostaining correlated with p63 immunostaining in seven of 10 cases. ${ }^{6,7,10-12,14,16}$ A strong association between purely cytoplasmic survivin localization and negative p63 staining was identified in five of seven cases. ${ }^{1-4,13}$ Furthermore, five of seven $\operatorname{cases}^{6,7,10,14,16}$ with metastasis or mortality on clinical follow-up, expressed p63. Therefore, our results support the previous observation that in Merkel cell carcinoma, p63 immunostaining is an independent prognostic marker for aggressive behavior. ${ }^{11}$ In addition, a strong correlation exists between nuclear survivin expression and p63 immunostaining in our case series, though limited by small case number. Proliferative index, as measured by MIB-1 (Ki-67) immunostaining did not correlate with survivin localization, p63 immunostaining, or clinical outcome in our series.

The prognostic significance of survivin immunohistochemical staining is unique because survivin is a powerful target for therapeutic intervention. Since survivin is specifically expressed in the tumor cells, but not in terminally differentiated human tissue it is an ideal target for anti-cancer therapy. Therefore, in contrast to other prognostic markers in tumors, survivin staining and localization may provide not only prognostic information, but also important information regarding the capacity for therapeutic intervention.

Numerous strategies are currently under investigation to identify potential mechanisms for anticancer therapy. Perhaps the most promising anticancer tool is derived from understanding the basic principles of survivin function. Survivin undergoes cell cycle-dependent phosphorylation
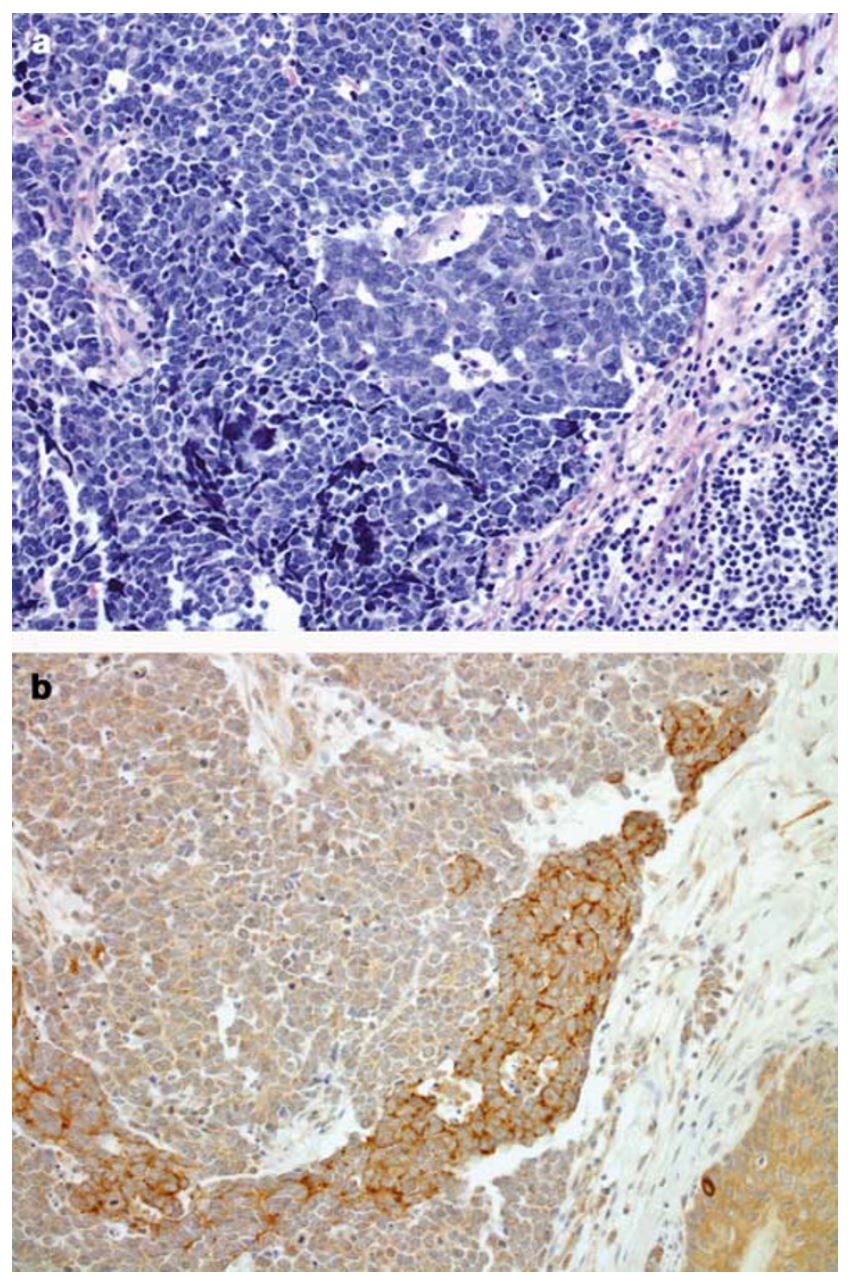

Figure 3 Large cell and small cell foci present within Merkel cell carcinoma. (a) Hematoxylin and eosin staining. (b) Survivin immunohistochemical staining showing strong cytoplasmic expression predominately in the larger cell component. Original magnification $\times 400$.

on T34 by then Cdc2/cyclinB1 complex. ${ }^{33}$ Phosphorylated survivin then forms an antiapoptotic complex with caspase 9 resulting in cellular survival. Inhibition of the Cdc2 kinase or mutation of the T34 residue to alanine (T34A) results in inhibition of the survivin-dependent antiapoptotic complex and therefore, promotes apoptosis. ${ }^{33}$ The mutation of specific phosphorylation sites whether in the regulation of apoptotic cytoplasmic function or nuclear mitotic function, is the first key to understanding the potential utility of survivin as an anticancer therapy. The major hurdle to overcome is the creation of an improved delivery system. To this end, fusion proteins that utilized the HIV TAT peptide sequence fused to either wild type or T34A mutant survivin were constructed and injected intraperitoneally into mice with subcutaneous melanoma xenografts. ${ }^{34}$ The T34A mutant fusion protein resulted in a $40-50 \%$ reduction in tumor growth and mass, as compared to wild type controls. $^{34}$ Therefore, the determination of the 
subcellular localization of survivin in tumors may be important clinically to predict prognosis and also therapeutically to link essential phosphorylation sites to the specific survivin function for mutant fusion protein construction.

In conclusion, survivin was expressed in all of our cases of Merkel cell carcinoma and the nuclear expression correlated with a poorer prognosis in our series. Though limited by small study number, these results may have diagnostic, prognostic, and therapeutic relevance to assist in the clinical management.

\section{Acknowledgement}

We are grateful to Vincent Klump for providing superb technical assistance.

\section{References}

1 Merkel F. Tastzellen und Taskörperchen bei den Hausthieren und beim Menschen. Arch Mikr Anat 1875;11:636.

2 Winkelman R, Breathnach A. The merkel cell. J Invest Dermatol 1973;60:2-15.

3 Toker C. Trabecular carcinoma of skin. Arch Dermatol 1972;105:107-110.

4 Miller R, Rabkin C. Merkel cell carcinoma and melanoma: etiological similarities and differences. Cancer Epidemiol Biomarkers Prev 1999;8:153-158.

5 Lemos B, Nghiem P. Merkel cell carcinoma: more deaths but still no pathway to blame. J Invest Dermatol 2007;127:2100-2103.

6 Goessling W, McKee P, Mayer R. Merkel cell carcinoma. J Clin Oncol 2002;20:588-598.

7 Coit D. Merkel cell carcinoma. Ann Surg Oncol 2001;9S:99S-102S

8 McAfee W, Morris C, Mendenhall C, et al. Merkel cell carcinoma: treatment and outcomes. Cancer 2005;104: 1761-1764.

9 Smith P, Patterson J. Merkel cell carcinoma (neuroendocrine carcinoma of the skin). Am J Clin Path 2001; 115S:S68-S78.

10 Skelton H, Smith K, Hitchcock C, et al. Merkel cell carcinoma: analysis of clinical histologic, and immunohistologic features of 132 cases with relation to survival. J Am Acad Dermatol 1997;37:734-739.

11 Asioli S, Righi A, Volante M, et al. p63 as a new prognostic marker in Merkel cell carcinoma. Cancer 2007;110:640-647.

12 Schlagbauer-Wadl H, Klosner G, Heere-Ress E, et al. Bcl-2 antisense oligonucleotides (G3139) inhibit Merkel cell carcinoma growth in SCID mice. J Invest Dermatol 2000;114:725-730.

13 Ambrosini D, Adida C, Altieri D. A novel antiapoptosis gene, survivin, expressed in cancer and lymphoma. Nat Med 1997;3:917-921.

14 Deveraux Q, Reed J. IAP family proteins suppressors of apoptosis. Genes Dev 1999;13:239-252.

15 Adida C, Crotty P, McGrath J, et al. Developmentally regulated expression of the novel cancer anti-apoptosis gene survivin in human and mouse differentiation. Am J Path 1998;152:43-49.
16 Kobayashi K, Hatano M, Otaki M, et al. Expression of a murine homologue of the inhibitor of apoptosis protein is related to cell proliferation. Proc Natl Acad Sci 1999;96:1457-1462.

17 Grossman D, McNiff J, Li F, et al. Expression and targeting of the apoptosis inhibitor, survivin, in human melanoma. J Invest Dermatol 1999;113:1076-1081.

18 Monzo M, Rosell R, Felip E, et al. A novel antiapoptosis gene: Re-expression of survivin messenger RNA as a prognosis marker in non-small-cell lung cancers. J Clin Oncol 1999;17:2100-2104.

19 Tanaka K, Iwamoto S, Gon G, et al. Expression of survivin and its relationship to loss of apoptosis in breast carcinomas. Clin Cancer Res 2000;6:127-134.

$20 \mathrm{Lu} \mathrm{C}$, Altieri D, Tanigawa N. Expression of a novel antiapoptosis gene, survivin, correlated with tumor cell apoptosis and p53 accumulation in gastric carcinomas. Cancer Res 1998;58:1808-1812.

21 Satoh K, Kaneko K, Hirota M, et al. Expression of survivin is correlated with cancer cell apoptosis and is involved in the development of human pancreatic duct cell tumors. Cancer 2001;92:271-278.

22 Kawasaki H, Altieri D, Lu C, et al. Inhibition of apoptosis by survivin predicts shorter survivial rates in colorectal cancer. Cancer Res 1998;58:5071-5074.

23 Saitoh Y, Yaginuma Y, Ishikawa M. Analysis of Bcl-2, Bax and Survivin genes in uterine cancer. Int J Oncol 1999;15:135-141.

24 Yoshida H, Ishiko O, Sumi T, et al. Survivin, bcl-2 and matrix metalloproteinase-2 enhance progression of clear cell- and serous-type ovarian carcinomas. Int J Oncol 2001;19:537-542.

25 Swana H, Grossman D, Anthony J, et al. Tumor content of the antiapoptosis molecule survivin and recurrence of bladder cancer. N Engl J Med 1999;341:452-453.

26 Piras F, Murtas D, Minerba L, et al. Nuclear survivin is associated with disease recurrence and poor survival in patients with cutaneous malignant melanoma. Histopathology 2007;50:835-842.

27 Bria E, Visca P, Novelli F, et al. Nuclear and cytoplasmic cellular distribution of survivin as survivial predictor in resected non-small-cell lung cancer. Eur J Surg Oncol 2007; doi:10.1016/j.ejso.2007.06.002.

28 Altieri D. Survivin, versatile modulation of cell division and apoptosis in cancer. Oncogene 2003; 22:8581.

29 Fortugno P, Wall N, Giodini A, et al. Survivin exists in immunohistochemically distinct subcellular pools and is involved in spindlemicrotubule function. J Cell Sci 2002;115:575-585.

30 Ruchaud S, Carmena M, Earnshaw W. Chromosomal passengers: conducting cell division. Nat Rev Mol Cell Biol 2007;8:798-812.

31 Adams R, Carmena M, Earnshaw W. Chromosomal passengers and the (aurora) ABCs of mitosis. Trends Cell Biol 2001;11:49-54.

32 Wheatley S, Barrett R, Andrews P, et al. Phosphorylation by aurora-B negatively regulates survivin function during mitosis. Cell Cycle 2007;6:1220-1230.

33 O'Connor D, Grossman D, Plescia J, et al. Regulation of apoptosis at cell division by p34cdc2 phosphorylation of survivin. Proc Natl Acad Sci 2000;97:13103-13107.

34 Yan $\mathrm{H}$, Thomas J, Lui T, et al. Induction of melanoma and inhibition of tumor growth using a cell-permeable Survivin antagonist. Oncogene 2006;25:6968-6974. 